\title{
Acknowledgements
}

The quality of our Journal depends on scholarly collaboration between the two groups of scholars, the authors and the anonymous peer-reviewers of their work. We thank both groups for their interest in and support of our Journal. We are especially grateful to the peer-reviewers listed below, who reviewed submissions to the current issue (Volume 7 Issue 1), for their time and commitment to excellent scholarship.

\section{Special Thanks to Our Reviewers}

Kathleen Absolon

Glen Aikenhead

Jo-Ann Archibald

Bonnie Freeman

Sheila Cote-Meek

Sara Davidson

Edward Doolittle

John Edwards
Isobel Findlay

Stephanie Inglis

Ruth Kolezar-Green

Marc Kuly

Victoria LaBillois

Naomi Metallic

Herman J. Michell

Nikki Moodie
Melissa Nelson

Michelle Pidgeon

Jean Paul Restoule

Michelle Sylliboy

Verna St. Denis

Yvone Vizina

Stanley Wilson

Pam Whitty

\section{Issue Statistics}

\section{A. Authors and Submissions}

\begin{tabular}{|l|l|}
\hline Authors and Co-Authors & \\
\hline University-based & 21 \\
\hline Non-University Based & 3 \\
\hline Total & $\mathbf{2 4}$ \\
\hline
\end{tabular}

\begin{tabular}{|l|r|}
\hline Article Submissions & \\
\hline Original proposals for peer and editor review & 29 \\
\hline Articles submitted for editor review & 3 \\
\hline Articles submitted for peer review & 12 \\
\hline Peer-reviewed articles accepted for publication & 10 \\
\hline Editor-reviewed articles accepted for publication & 2 \\
\hline Book reviews submitted for editor review & 1 \\
\hline Book reviews accepted for publication & 1 \\
\hline
\end{tabular}




\begin{tabular}{|l|l|}
\hline Geographic Distribution (Corresponding Authors Only) & \\
\hline Atlantic Canada & \\
\hline Cape Breton University & 1 \\
\hline Non-University Based & 1 \\
\hline & \\
\hline Eastern Canada & \\
\hline Institut National de la Recherche Scientifique & 1 \\
\hline Wilfrid Laurier University & 1 \\
\hline & \\
\hline Western Canada & \\
\hline Royal Roads University & 1 \\
\hline Simon Fraser University & 2 \\
\hline University of Alberta & 1 \\
\hline University of Calgary & 1 \\
\hline University of Saskatchewan & 2 \\
\hline & \\
\hline International & \\
\hline Griffith University, Australia & 1 \\
\hline University of Melbourne, Australia & 1 \\
\hline University of New Mexico, USA & 1 \\
\hline & \\
\hline Total & \\
\hline
\end{tabular}

\section{B. Peer-Reviewers and Peer-Reviewing}

\begin{tabular}{|l|r|}
\hline Peer Reviewers & \\
\hline Total invitations to peer review & 51 \\
\hline Number of peer reviewers who accepted invitations & 24 \\
\hline
\end{tabular}

\begin{tabular}{|l|r|}
\hline Geographic Distribution (Peer Reviewers) & \\
\hline Atlantic Canada & 1 \\
\hline Cape Breton University & 1 \\
\hline Dalhousie University & \\
\hline
\end{tabular}




\begin{tabular}{|l|r|}
\hline St. Francis Xavier University & 1 \\
\hline University of New Brunswick & 1 \\
\hline & \\
\hline Eastern Canada & 1 \\
\hline McMaster University & 1 \\
\hline Wilfrid Laurier University & 2 \\
\hline York University & \\
\hline & 1 \\
\hline Western Canada & 2 \\
\hline First Nations University & 1 \\
\hline Simon Fraser University & 1 \\
\hline University of Alberta & 1 \\
\hline University of British Columbia & 3 \\
\hline University of Fraser Valley & 1 \\
\hline University of Saskatchewan & 2 \\
\hline University of Victoria & \\
\hline University of Winnipeg & 2 \\
\hline & \\
\hline Non-University Based & 1 \\
\hline
\end{tabular}

